\title{
Measurements of the Higgs Boson at the LHC and Tevatron
}

\author{
Somnath Choudhury ${ }^{1, a, b}$ \\ ${ }^{1}$ DESY - Hamburg, Germany
}

\begin{abstract}
The results on the observation of the Higgs boson in decays to bosons and fermions have been reported from the LHC and Tevatron. A direct evidence of the Higgs-lepton coupling is established at the LHC with the tau pair decay mode and a direct indication of Higgs boson coupling to fermions is observed in decays to bottom quarks at the Tevatron. The measurements of the Higgs boson mass, coupling to vector bosons and fermions, spin and parity, decay width and production cross section have been reported.
\end{abstract}

\section{Introduction}

The ATLAS and CMS collaborations at the LHC in July 2012 came out with the observation of a Higgs boson [1, 2] using proton-proton collision data from the LHC at CERN corresponding to integrated luminosities of around $5 \mathrm{fb}^{-1}$ at $7 \mathrm{TeV}$ and $5 \mathrm{fb}^{-1}$ at $8 \mathrm{TeV}$ center-of-mass energies. The LHC has completed its first run with the detectors having recorded about $95 \%$ of delivered collision data, among which about $90 \%$ were certified and used to obtain the results reported here. The final analysis is based on integrated luminosity of $20 \mathrm{fb}^{-1}$ at $8 \mathrm{TeV}$ in addition to the $5 \mathrm{fb}^{-1}$ at $7 \mathrm{TeV}$ collected by the ATLAS [3] and CMS detectors [4] at the LHC. The observation of a Higgs boson with a mass of about $125 \mathrm{GeV}$ in the high resolution boson decay channels motivates the search in major fermion decay modes as decays of the Higgs boson to tau pairs and bottom quark pairs have significantly large branching fraction in this mass regime. The $125 \mathrm{GeV}$ Higgs boson opens an interesting avenue for its decays to fermions as this would provide an effective handle for the measurement of the Higgs boson coupling to fermions. The DØ and CDF experiments presented a combined result from the Tevatron using around $10 \mathrm{fb}^{-1}$ of proton-antiproton collision data at $1.96 \mathrm{TeV}$ showing an excess of events in the bottom quark decay mode in association with vector bosons which is compatible with the presence of the $125 \mathrm{GeV}$ Higgs boson observed by ATLAS and CMS experiments at the LHC.

The LHC experiments had to establish methods to cope with the challenge of a high number of multiple collisions per beam crossing (pileup), which occurs at high luminosity. The successful mitigation of pileup was demonstrated and an almost uniform response of the missing transverse energy resolution as a function of the number of primary vertices in the event is achieved. The standard model (SM) Higgs boson analyses utilise the major Higgs

\footnotetext{
${ }^{\mathrm{a}}$ for the ATLAS, CMS, CDF and DØ collaborations

be-mail: somnathchoudhury@gmail.com
}

boson production mechanisms in proton-proton collisions which are in order of decreasing cross sections namely gluon fusion, vector boson fusion (VBF) and associated production with vector bosons $(\mathrm{VH})$. A measurement of the rate of Higgs boson production in association with top quark pair provides a direct test of the coupling between the top quark and the Higgs boson and has also been studied by the ATLAS and CMS experiments.

The measurement of the properties of the discovered Higgs boson is crucial to understand the nature of the new particle and study its compatibility with the standard model. Any deviations from SM expectations would definitely indicate towards new physics at the $\mathrm{TeV}$ scale signalling scenarios beyond the standard model such as supersymmetry. The mass of the Higgs boson has been measured in the high resolution boson decay modes, $\mathrm{H} \rightarrow \gamma \gamma$ and $\mathrm{H} \rightarrow \mathrm{ZZ}^{*} \rightarrow 4 \ell$, at the LHC. The couplings of the Higgs boson with respect to the SM expectations and the spin and parity of the new particle have also been measured with several spin/parity hypothesis tested and ruled out except for the scalar boson. Moreover, direct constraints on the Higgs boson decay width with a high level precision of the order of $20 \mathrm{MeV}$ and measurement of the Higgs boson fiducial and differential production cross sections have been reported from the LHC.

\section{The Colliders and Associated Detectors}

The Tevatron was a proton-antiproton collider at Fermilab which completed operations in September 2011. The Higgs boson searches took place during the Tevatron Run II phase (2002-2011), in which it was configured to collide beams of 36 bunches with $1.96 \mathrm{TeV}$ center-of-mass energy and provided an integrated data set of $10 \mathrm{fb}^{-1}$ to the CDF and DØ experiments (note that integrated luminosities given here refers to integrated luminosity delivered with the detectors in an operational condition sufficient to be used for physics analysis). The instantaneous luminosity reached $4 \times 10^{32} \mathrm{~cm}^{2} \mathrm{~s}^{-1}$, but the effect of the overlay of 
multiple interactions remained under control. The primary components of the Run-II CDF [5] and DØ [6] detectors are the silicon and fiber tracking detectors immersed in a solenoidal magnetic field, electromagnetic and hadronic calorimeters, and drift chambers for the muon detection system. The kinematic properties of particles and jets produced in the collision events are defined with respect to the origin of the detector coordinate system which is at the center of the detector. To quantify polar angles the pseudorapidity variable, defined as $\eta=-\ln (\theta / 2)$, is used where $\theta$ is the polar angle in the spherical polar coordinate system.

The LHC accelerator is a proton-proton collider at CERN operating at the highest energies currently attained by a hadron collider, which started operation in 2010. During the 2011 data taking period it was configured to collide beams with $7 \mathrm{TeV}$ center-of-mass energy and provided an integrated data set of just over $5 \mathrm{fb}^{-1}$ to the LHC experiments, while during 2012 it was configured at $8 \mathrm{TeV}$ and provided an integrated data set of approximately $20 \mathrm{fb}^{-1}$ to ATLAS and CMS. The instantaneous luminosity of the LHC has reached $7.7 \times 10^{33} \mathrm{~cm}^{2} \mathrm{~s}^{-1}$ making 20-30 multiple interactions per crossing a typical occurrence and creating additional challenges for triggering on and reconstructing physics events. A proton-proton collider at high energy provides large cross sections for gluon-gluon or quarkquark initiated Higgs boson production processes such as gluon fusion and vector boson fusion. For instance, the cross section for gluon fusion to a Higgs boson is increased by a factor of approximately 15 compared to the Tevatron. In addition, the increase in center-of-mass energy from 7 to $8 \mathrm{TeV}$ correspondingly raises the Higgs boson cross section by an additional factor of approximately $30 \%$.

The LHC experiments are forward-backward and cylindrically symmetric detectors with tracking, calorimetric and muon detector elements. The ATLAS detector includes an inner tracking and vertexing system, electromagnetic and hadronic calorimetry, and an outer muon detection system. The inner tracking detector consists of a silicon pixel detector, a silicon microstrip detector and a transition radiation tracker immersed in the field of a $2 \mathrm{~T}$ solenoidal magnet which provides charged particle tracking and vertex finding over a large pseudorapidity range of $|\eta|<2.5$. The inner tracker and solenoid are surrounded by a high-granularity liquid-argon sampling electromagnetic calorimeter which provides electron (photon) reconstruction in the pseudorapidity range $|\eta|<2.47(|\eta|<2.37)$. An iron-scintillator tile calorimeter provides hadronic coverage in the central rapidity range. The endcap and forward regions are instrumented with liquid-argon calorimetry for both electromagnetic and hadronic measurements which extends jet finding to forward rapidity of $|\eta|<4$.9. The muon spectrometer surrounds the calorimeters and consists of three large superconducting toroids, each with eight coils, a system of precision tracking chambers and detectors for triggering in the range $|\eta|<2.4$.

The CMS detector consists of a barrel assembly and two endcaps, comprising, in successive layers outwards from the collision region, a silicon pixel and strip tracker, a lead tungstate crystal electromagnetic calorimeter, a brass scintillator hadron calorimeter, a $3.8 \mathrm{~T}$ superconducting solenoid, and gas-ionization chambers embedded in the steel return yoke for the detection of muons. The silicon detector provides charged particle tracking and vertexing for b-tagging over a large pseudorapidity range of $|\eta|<2.5$, which is well matched to the coverage of the barrel and electromagnetic calorimeter as well as that of the muon chambers providing coverage to $|\eta|<3$ and $|\eta|<2.4$ for electron and muon identification, respectively. The return field of the magnet allows independent momentum measurement and triggering in the muon chambers. The identification of photons and $\tau$ leptons is performed within the overlapping pseudorapidity range of the tracker and electromagnetic calorimeter. Jet finding can be performed in an expanded pseudorapidity range up to $|\eta|<5.0$ using forward calorimeters. For the physics object reconstruction in a collision event, CMS uniquely employs a particle flow technique which provides event description in the form of mutually exclusive particles identifying all stable particles produced in the event by combining the capabilities of each sub-detector with the most precise measurement of the energy and direction for each particle and then individual measurements from each sub-detector are combined by a geometrical linking algorithm providing particle identification on blocks of these linked elements.

\section{Higgs Boson at the LHC and Tevatron}

Both ATLAS and CMS detectors use a multilevel triggering system. The first level of the trigger allows for the measurement of the momentum or energy of physics objects including electrons and photons as electromagnetic energy deposits, muons with independent measurement of the momentum in the muon systems, jets and missing transverse energy using full calorimeter information, and taus as narrow jets. The event rate is reduced to approximately $100 \mathrm{kHz}$ at level one. The ATLAS experiment employs a second level trigger which repeats physics object identification using full granularity of the detector and further reduces the event rate to $3.5 \mathrm{kHz}$. Both detectors employ a full event reconstruction using optimized versions of offline reconstruction software running on commercial processors as level 3 or high level event filter with an output rate below $400 \mathrm{~Hz}$ for ATLAS and $500 \mathrm{~Hz}$ for CMS.

The CDF trigger system consists of three levels. Level one trigger hardware consists of dedicated electronics that operate at the beam crossing frequency. The level one trigger can identify and measure the transverse momentum of charged particles and combine with information from the calorimeters or muon systems to provide a trigger for leptons. The calorimeter trigger hardware measures energy clusters which are used to identify jets and photons as well as an imbalance in event transverse energy. The second level trigger hardware at CDF refines the measurements of the level one trigger at higher precision. The level two trigger can also include tracking and vertexing information from the silicon detectors. The third level of the trigger operates on commercial computers and executes fast versions of the full offline reconstruction software. 

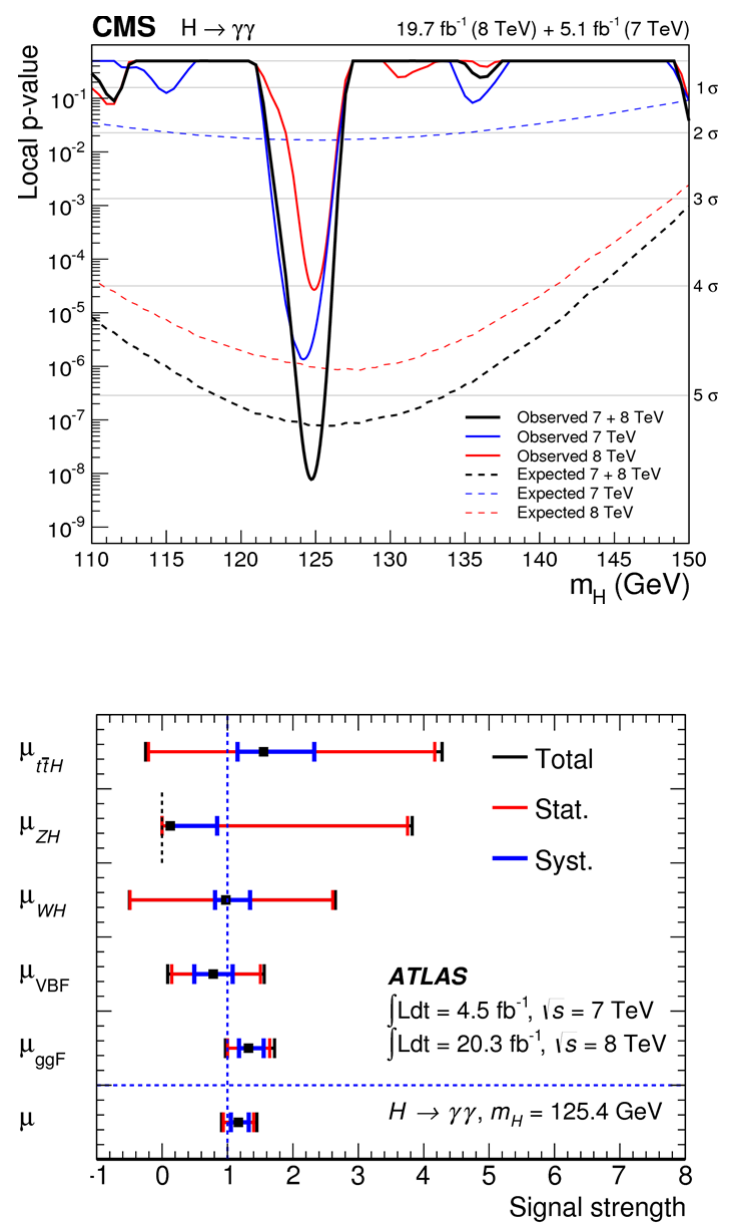

Figure 1. The Higgs boson signal at $125 \mathrm{GeV}$ with significance over $5 \sigma$ observed in the di-photon decay mode in CMS (top) [9] and the measured Higgs boson signal strength at $\mathrm{m}_{\mathrm{H}}=125.4 \mathrm{GeV}$ for the di-photon decay channel in different production modes in ATLAS (bottom) [7].

The $\mathrm{D} \varnothing$ trigger system also has three trigger levels referred to as L1, L2, and L3. Each consecutive level receives a lower rate of events for further examination. The L1 hardware based elements of the triggers used in the electron channel typically require calorimeter energy signatures consistent with an electron. This is expanded at L2 and L3 to include trigger algorithms requiring an electromagnetic object together with at least one jet for which the $\mathrm{L} 1$ requirement is calorimeter energy depositions consistent with high- $\mathrm{p}_{\mathrm{T}}$ jets. For muon samples, events are triggered using the logical OR of the full list of available triggers of $\mathrm{D} \varnothing$. Events not selected by high- $\mathrm{p}_{\mathrm{T}}$ muon triggers (for majority of $\mathrm{W}+$ jet events are collected by high- $\mathrm{p}_{\mathrm{T}}$ muon triggers) are primarily collected by the jet triggers.

\subsection{Higgs Boson in Bosonic Decays}

$\mathbf{H} \rightarrow \gamma \gamma$

The LHC experiments have the ability to reconstruct a Higgs boson in the two photon decay mode [7, 9] as a narrow peak over a smoothly falling background. The detectors are designed with the excellent energy and position

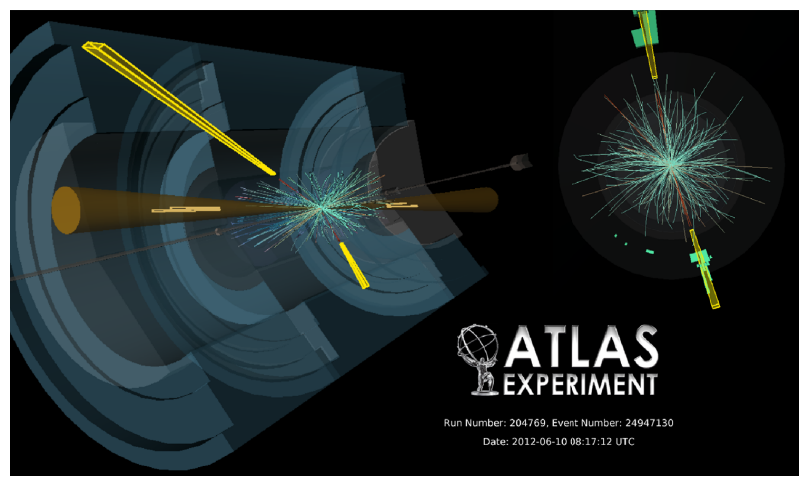

Figure 2. A typical ATLAS event display of the Higgs boson decaying to two photons (in yellow) in the vector boson production mode with two forward jets (in brown) [8].

resolution necessary to accurately reconstruct the invariant mass of two photon events. Excellent mass resolution is critical since background contributions from multijet, multijet + photon, and di-photon events are huge. This decay has a small branching ratio of $0.2 \%$, since the Higgs boson can decay only to photons through a loop diagram involving massive charged particles. The large inclusive production cross section at lower Higgs boson masses makes this a viable mode for a Higgs boson observation at low mass. In addition, observation of this mode rules out spin one for the observed particle. Energy and isolation requirements are imposed on the photons to reduce the backgrounds. Converted photons are also used and provide good energy and position resolution when electron pairs can be reconstructed by the trackers. The ATLAS experiment reduces background by using the longitudinal segmentation of the calorimeters to select photons that point to the hard interaction vertex. Both experiments apply a separate selection for events with two forward jets to focus on a vector boson fusion like topology which has excellent sensitivity achieving a signal to background that is an order of magnitude greater than in topologies without forward jets typical of gluon fusion production. In the ATLAS experiment, to optimize sensitivity, events are further divided by whether photons are converted or not and by which pseudorapidity region of the detector they are reconstructed in, since these classes result in different diphoton mass resolution and as a result different signal to background ratio. Finally, events are classified by the transverse momentum of the diphoton system since the backgrounds are substantially reduced at high values. In the CMS experiment, the output of a dedicated photon identification boosted decision tree (BDT), the transverse momentums of the photons, the opening angle between the photons, the pseudorapidity of the photons, and the estimated event-by-event mass resolutions of the diphoton system are used to classify events by expected signal-to-background ratios using a BDT multivariate discriminate. An investigation of the categories formed by selecting on the BDT output indicates that divisions based on the transverse momentum of the diphoton system, the detector pseudorapidity region of the photons, and 
whether the photons are converted or unconverted largely determines the classification of events. The sensitivities of the individual categories are taken into account when calculating the overall sensitivity of the analysis and when determining exclusions or signal significances. The events are weighted by expected signal-to-background ratio when displaying the diphoton mass distribution to give a visual representation of the benefit of this classification. Using the two photon invariant mass distribution to search for the Higgs boson, regions at both low mass and high mass have been excluded by both LHC experiments leaving only a narrow region of mass unexcluded.

The ATLAS experiment extends the lower exclusion bound of the LHC searches below the upper exclusion bound from the LEP searches, and when combined with other searches excludes the entire mass range below $600 \mathrm{GeV}$ except for the narrow allowed region around $125 \mathrm{GeV}$. The number of observed Higgs boson decays to diphotons divided by the corresponding SM prediction, called the signal strength, is found to be $\mu=1.17 \pm 0.27$ at the value of the Higgs boson mass measured by ATLAS, $\mathrm{m}_{\mathrm{H}}=125.4 \mathrm{GeV}$. The analysis is optimized to measure the signal strengths for individual Higgs boson production processes at this value of $\mathrm{m}_{\mathrm{H}}$. They are found to be $\mu_{\mathrm{ggF}}=1.32 \pm 0.38, \mu_{\mathrm{VBF}}=0.8 \pm 0.7, \mu_{\mathrm{WH}}=1.0 \pm 1.6$, $\mu_{\mathrm{ZH}}=0.1_{-0.1}^{+3.7}, \mu_{\mathrm{t} \mathrm{tH}}=1.6_{-1.8}^{+2.7}$, for Higgs boson production through gluon fusion, vector-boson fusion, and in association with a $\mathrm{W}$ or $\mathrm{Z}$ boson or a top quark pair, respectively as seen in Figure 1. Compared with previous ATLAS analyses, the results reported here also benefit from a new energy calibration procedure for photons and the subsequent reduction of the systematic uncertainty on the diphoton mass resolution. A clear signal is observed by the CMS experiment in the diphoton channel as observed in Figure 1 at a mass close to $125 \mathrm{GeV}$ with a local significance of $5.7 \sigma$, where a significance of $5.2 \sigma$ is expected for the standard model Higgs boson. The mass is measured to be $124.70 \pm 0.34 \mathrm{GeV}=124.70 \pm 0.31$ (stat) \pm 0.15 (syst) $\mathrm{GeV}$, and the best-fit signal strength relative to the SM expectations is $1.14_{-0.23}^{+0.26}=1.14 \pm 0.21$ (stat) ${ }_{-0.05}^{+0.09}$ (syst) ${ }_{-0.09}^{+0.13}$ (theory). No significant deviations from the predictions of the standard model are found by both ATLAS and CMS.

$\mathbf{H} \rightarrow \mathrm{ZZ}^{*} \rightarrow \mathbf{4} \ell$

The $\mathrm{H} \rightarrow \mathrm{ZZ}^{*} \rightarrow 4 \ell$ decay mode $[10,12]$ at the $\mathrm{LHC}$ is considered as the golden channel as it is a very powerful search channel that is sensitive to a SM Higgs boson throughout its entire allowed mass range with a clean signature of four isolated leptons from a common primary vertex. The analysis focuses on the electron and muon final states where the 4-lepton invariant mass resolution is excellent and the background due to jet fakes is strongly suppressed. The major backgrounds to the signal include the di-boson $\mathrm{ZZ}, \mathrm{Z}+$ jets and top pair production. The SM di-boson irreducible ZZ background is estimated from Monte Carlo simulation. The $\mathrm{Z}+$ jets and top backgrounds are estimated from control samples in data. In such background estimation method, a control region is defined by

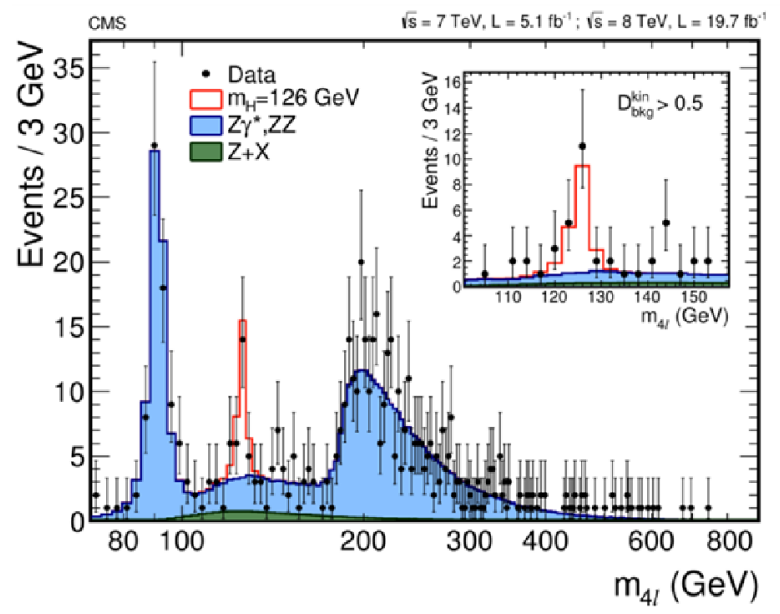

Figure 3. The 4-lepton invariant mass distribution in the CMS experiment clearly showing the Higgs boson mass peak over an almost flat $\mathrm{ZZ}$ background in the mass range of interest [12].

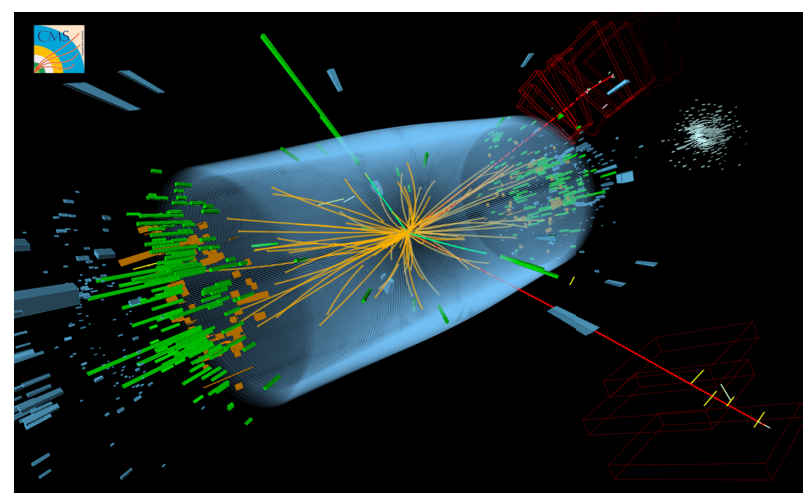

Figure 4. A typical CMS event display of the Higgs boson decaying to four leptons with 2 muons (in red) and 2 electrons (in green) as final state signatures.

reversing at least one signal selection requirement, and additional criteria can be added so that the control sample has a particular type of background contribution enhanced and is not overlapped with the signal sample where all events passed the final selection. The background normalization in the control sample can be related to its normalization in the signal sample via a transfer factor. This transfer factor and its uncertainty are usually determined from the simulation samples. The background contribution in the signal sample can therefore be determined by the product of the background contribution in the data control sample and the transfer factor. Such method provides a data driven estimation of the background, and is widely used in the LHC experiments, including most Higgs boson related analyses. The selected events are classified to $4 \mu, 2 \mu 2 \mathrm{e}$ and $4 \mathrm{e}$ categories, depending on the flavour of the leading pair of leptons. Events are further classified to enhance the sensitivity to various Higgs boson production modes.

The final Run-I measurements of Higgs boson production and couplings in the decay channel $\mathrm{H} \rightarrow \mathrm{ZZ}^{*} \rightarrow 4 \ell$ are presented. At CMS, the new boson is observed as a narrow resonance as seen in Figure 3, with a local sig- 
nificance of 6.8 standard deviations, a measured mass of $125.6 \pm 0.4$ (stat.) \pm 0.2 (syst.) $\mathrm{GeV}$, and a total width less than $3.4 \mathrm{GeV}$ at a $95 \%$ confidence level. The limits at CMS are derived using a fit of the 4-lepton invariant mass distribution with a kinematic discriminant constructed out of the angular decay variables of the 4lepton system and the properties sensitive to the production mechanism. The production cross section of the new boson times the branching fraction to four leptons is measured to be $0.93_{-0.23}^{+0.26}(\text { stat. })_{-0.09}^{+0.13}$ (syst.) times that predicted by the standard model. In the ATLAS experiment, the $\mathrm{H} \rightarrow \mathrm{ZZ}^{*} \rightarrow 4 \ell$ signal is observed with a significance of 8.1 standard deviations at $125.36 \mathrm{GeV}$, the combined ATLAS measurement of the Higgs boson mass from the $\mathrm{H} \rightarrow \gamma \gamma$ and $\mathrm{H} \rightarrow \mathrm{ZZ}^{*} \rightarrow 4 \ell$ channels. The production rate relative to the standard model expectation, the signal strength, is measured in four different production categories in the $\mathrm{H} \rightarrow \mathrm{ZZ}^{*} \rightarrow 4 \ell$ channel. The measured signal strength, at this mass, and with all categories combined, is $1.44_{-0.33}^{+0.40}$. The signal strength for Higgs boson production in gluon fusion or in association with $t \bar{t}$ or $b \bar{b}$ pairs is found to be $1.7_{-0.4}^{+0.5}$, while the signal strength for vector boson fusion combined with $\mathrm{WH} / \mathrm{ZH}$ associated production is found to be $0.3_{-0.9}^{+1.6}$.

\subsection{Higgs Boson in Fermionic Decays}

$\mathbf{H} \rightarrow \tau \tau$

The $\mathrm{H} \rightarrow \tau \tau$ search $[13,14]$ at the LHC is performed using all possible decays in fully leptonic, semi-leptonic and fully hadronic states using the final-state signatures $\mathrm{e} \mu$, $\mu \mu$, ee, e $\tau_{h}, \mu \tau_{h}$ and $\tau_{h} \tau_{h}$, where electrons and muons arise from leptonic $\tau$-decays and $\tau_{h}$ denotes hadronic tau lepton decays. To enhance the sensitivity of the search at the CMS experiment, each of these categories is further divided into three exclusive sub-categories according to the nature of the associated jets in the event. The gluon-fusion production mechanism has the largest production cross section. However, in the mass region of interest, background from Drell-Yan production of tau pairs overwhelms the expected Higgs boson signal. This search therefore relies strongly upon the signature of Higgs bosons produced via vector boson fusion (VBF) or in association with a high transverse momentum $\left(\mathrm{p}_{\mathrm{T}}\right)$ jet recoiling against the tau pair. In the former case, the distinct topology of two jets with a large rapidity separation greatly reduces the background. In the latter, requiring a high- $\mathrm{p}_{\mathrm{T}}$ jet both suppresses background, and improves the resolution of the tau-pair invariant mass. The CMS search has been categorized into 0 -jet, 1 -jet and 2-jet VBF categories to extract the signal which are further split on the basis of hadronic tau $\mathrm{p}_{\mathrm{T}}$ and di-tau $\mathrm{p}_{\mathrm{T}}$ for 0 and 1 jet categories or di-tau $\mathrm{p}_{\mathrm{T}}$, di-jet invariant mass and dijet pseudorapidity separation for 2 -jet category. The 0 -jet category constrains the background normalization, identification efficiencies and energy scales, the 1-jet category improves the resolution of Higgs boson mass and the VBF category has high signal over background ratio. The analysis is also performed in the VH category with lepton tag-
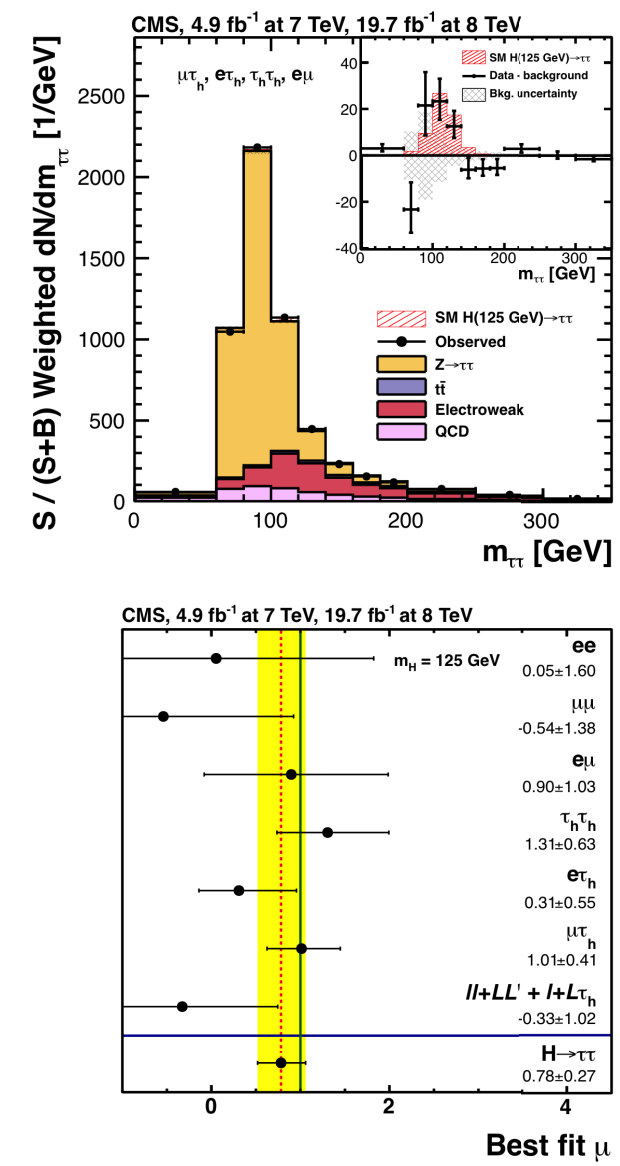

Figure 5. The di-tau invariant mass distribution combining all categories (top) and the signal strength with respect to SM expectation in different di-tau decay channels and result of the combination in CMS (bottom) [14].

ging from vector bosons along with the tau lepton pair. The ATLAS experiment also uses similar approach classifying events in VBF (2-jet) and Boosted categories, where the boosted category has high $\mathrm{p}_{\mathrm{T}}$ di-tau events. The CMS experiment distinguishes the Higgs boson signal from the background from the tau-pair invariant mass reconstructed using a maximum likelihood technique. The algorithm estimates the original momentum components of the two taus by maximizing a likelihood with respect to free parameters corresponding to the missing neutrino momenta, subject to kinematic constraints. Other terms in the likelihood take into account the tau-decay phase space and the probability density in the tau transverse momentum, parametrized as a function of the tau-pair mass. This algorithm yields a tau-pair mass with a mean consistent with the true value, and distribution with a nearly Gaussian shape. The relative $\mathrm{m}_{\tau \tau}$ mass resolution estimated from simulation is $10-20 \%$ depending on the di-tau decay channel and category. The likelihood based mass reconstruction allows for a better separation between simulated $125 \mathrm{GeV}$ Higgs boson signal and $\mathrm{Z} \rightarrow \tau \tau$ background than the visible mass alone, yielding an improvement in the final expected significance of $40 \%$. For tau pair mass reconstruction ATLAS uses an algorithm called the Miss- 

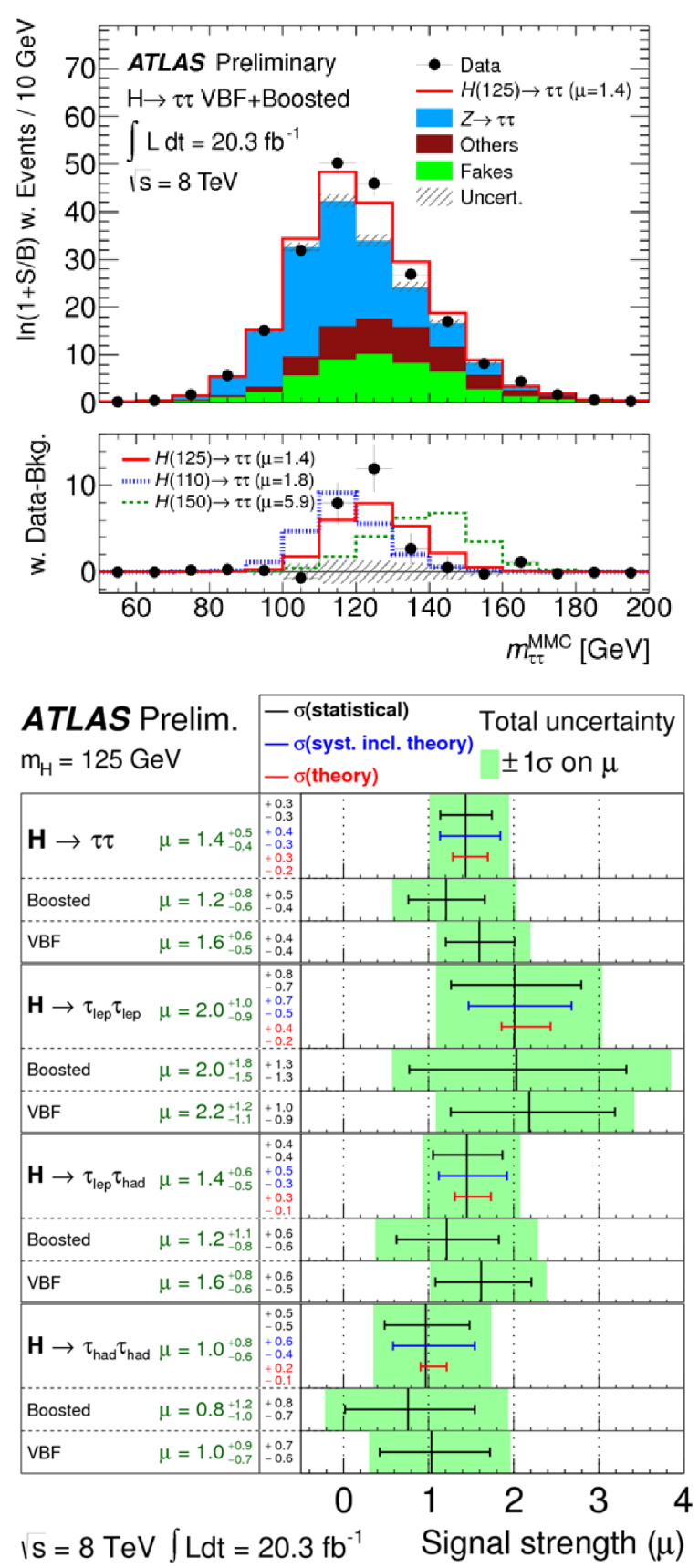

Figure 6. The di-tau invariant mass distribution combining all categories (top) and the signal strength with respect to the SM expectation in different di-tau decay channels and result of the combination in ATLAS (bottom) [13].

ing Mass Calculator, which provides an accurate mass reconstruction of the original resonance and does not suffer from the limitations of the collinear approximation. The major improvement comes from replacing assumptions of the collinear approximation by a requirement that mutual orientations of the neutrinos and other visible decay products are consistent with the mass and decay kinematics of a tau lepton. This is achieved by maximizing a probability defined in the kinematically allowed phase space region. It drops any assumption of collinearity and tries to resolve the equations of the missing transverse energy
(MET) and the tau lepton masses in terms of the neutrinos momenta, and for each possible solution a reconstructed mass is created that is weighted by the output of a likelihood function. The MET mismeasurement is taken into account by the use of an extra term in the likelihood, basically parametrizing the MET uncertainty. The estimator for the tau pair mass is then defined as the most probable value of the weighted scan points. The largest source of irreducible background to the search is $Z \rightarrow \tau \tau$ which is estimated using an observed sample of $\mathrm{Z} \rightarrow \mu \mu$ events, where the reconstructed muons are replaced by the reconstructed particles from simulated tau decays, a procedure called "embedding". The normalization for this process is determined from the measurement of the CMS measured cross section. The reducible backgrounds ( $\mathrm{W}+\mathrm{jets}$, multijet production, $\mathrm{Z}+$ jets) are also evaluated from control samples in data. The QCD multijet background is evaluated using the ratio of opposite-sign (OS) to same-sign (SS) di-tau events and relaxed lepton isolation after an estimate of the $\mathrm{W}+$ jets background using the high transverse mass $\left(\mathrm{m}_{\mathrm{T}}{ }^{\ell E_{\mathrm{T}}^{\text {miss }}}\right)$ side-band $\mathrm{W}$ boson enriched region and extrapolating it to the signal region. The $\mathrm{Z}+$ jets background is evaluated from fake rate and OS/SS ratio with the shape from simulation. The top pair produced events and diboson contribution are estimated using simulation.

The ATLAS experiment used a Boosted Decision Tree (BDT) approach, trained and validated separately in each channel and category before being fit simultaneously to extract signal. After boosting, the final score undergoes a monotonic transformation to spread the scores between -1 and 1 . The most signal-like events have scores near 1 while the most background-like have scores near -1 . The CMS experiment used the tau pair invariant mass as the discriminating variable. Both ATLAS and CMS see an excess of events over the background prediction. At CMS using the full Run-I data set, combining all event categories, a broad excess of events is observed in the tau pair invariant mass distribution as seen in Figure 5 over a range of the Higgs boson mass consistent with the $125 \mathrm{GeV}$ scalar boson observed in the high resolution boson decay channels. The observed (expected) significance of the excess at Higgs boson mass of $125 \mathrm{GeV}$ is $3.2 \sigma(3.7 \sigma)$. The best-fit value of the signal strength is $\mu=0.78 \pm 0.27$, obtained in the global fit combining all channels included in this analysis where the $\mathrm{H} \rightarrow \mathrm{WW}$ process has been added as a background for the observed process. In the ATLAS experiment, with $20.3 \mathrm{fb}^{-1}$ of $8 \mathrm{TeV}$ data, the observed (expected) deviation from the background-only hypothesis corresponds to a significance of 4.1 (3.2) standard deviations, and the measured signal strength is $\mu=1.4_{-0.4}^{+0.5}$ as in Figure 6. These results provide the first direct indication of the Higgs boson coupling to leptons.

\section{$\mathbf{H} \rightarrow \mathbf{b} \overline{\mathbf{b}}$}

The major decay mode for the Higgs boson at the Tevatron at a mass of $125 \mathrm{GeV}$ is $\mathrm{H} \rightarrow \mathrm{b} \bar{b}$ [16] with $58 \%$ branching ratio. The most sensitive signatures in this decay are: one lepton + missing $\mathrm{E}_{\mathrm{T}}+$ two b-jets $(\mathrm{WH} \rightarrow \ell \nu \mathrm{b} \bar{b})$, no lepton 


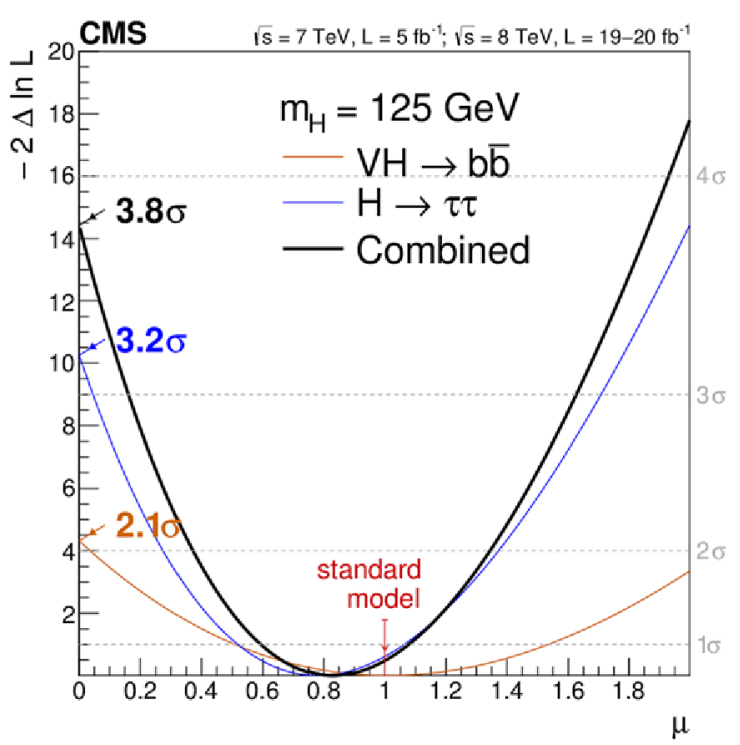

Figure 7. Scan of the profile likelihood as a function of the signal strength relative to the expectation for the production and decay of SM Higgs boson for $\mathrm{m}_{\mathrm{H}}=125 \mathrm{GeV}$ at CMS [15]. The statistical significance against the background-only hypothesis is shown for the two channels and their combination.

+ missing $\mathrm{E}_{\mathrm{T}}+$ two b-jets $(\mathrm{ZH} \rightarrow v \bar{v} \mathrm{~b} \overline{\mathrm{b}})$ and two leptons + two b-jets $\left(\mathrm{ZH} \rightarrow \ell^{+} \ell^{-} \mathrm{b} \overline{\mathrm{b}}\right)$. Over the Tevatron Run-II period, both $\mathrm{CDF}$ and $\mathrm{D} \emptyset$ have followed the same strategy to optimize the analyses $[17,18]$ and improve their sensitivity faster than expected from just accumulating more data. Acceptance is maximized by lowering kinematic requirements on leptons, by including different lepton reconstruction categories and by accepting events from all possible triggers. MVA techniques are widely used in all channels as they provide typically $25 \%$ more sensitivity than just using single kinematic discriminant such as the dijet mass for the $\mathrm{VH} \rightarrow \mathrm{Vb} \bar{b}$ channels. Each MVA combines into a single discriminant many variables, which include for example variables describing the event topology, the lepton and jet kinematics, the quality of leptons, and the relation between leptons/jets and missing transverse energy. The MVA techniques are also employed for object identification (b-jets, leptons, photons) and for energy correction to b-jets. Another way of achieving better sensitivity to signal, consists in splitting the search channels into sub-channels according to jet multiplicity, b-tagging content, lepton flavour or lepton quality. Dedicated MVA are also trained to split analyses into subchannels enhanced or enriched in specific backgrounds. Using sub-channels with different signal-over-background ratio $(\mathrm{s} / \mathrm{b})$ maximizes discriminating power, allows sensitivity to different signal production modes, and gives more handles to control backgrounds and systematic uncertainties. Data driven methods have been employed to control backgrounds and validate search methods. Instrumental backgrounds, such as jets faking leptons, photons faking electrons, charge mismeasurements, and tail of missing transverse resolution are measured in dedicated control samples. Background enriched samples are also employed
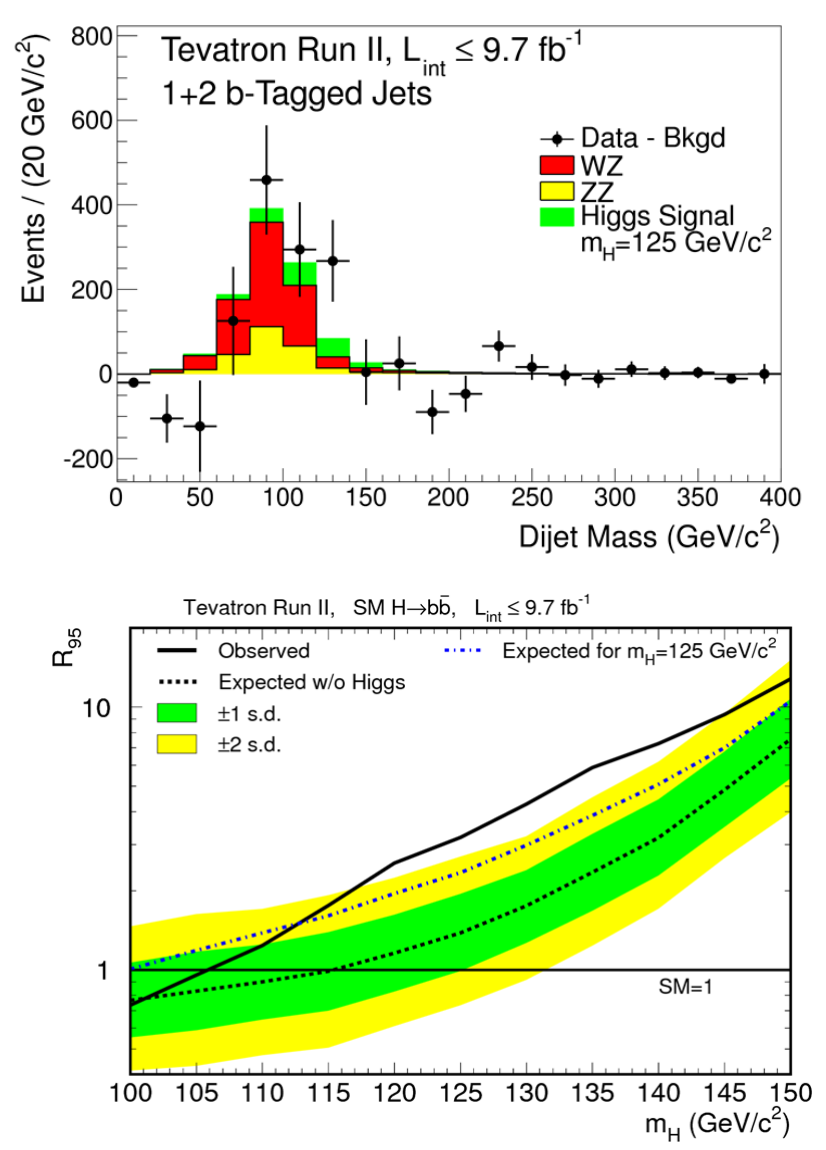

Figure 8. The Tevatron background-subtracted distribution of di-jet mass in $\mathrm{VH} \rightarrow \mathrm{Vb} \bar{b}$ channel summed over all input channels (top) and the observed 95\% C.L. upper limits on SM Higgs boson production as a function of Higgs boson mass (bottom) [16].

to check modeling of specific background processes. A broad excess of events is observed over a range of Higgs boson mass from 115 to $140 \mathrm{GeV}$ with a global significance of $3.1 \sigma$ as seen in Figure 8, which is consistent with the observed boson at $125 \mathrm{GeV}$ at the LHC. The significance of the excess at $125 \mathrm{GeV}$ is found to be $2.8 \sigma$ providing direct indication of Higgs boson coupling to fermions.

\subsection{Higgs Boson Production with Top Quarks}

In the search for Higgs boson production in association with top quarks $[19,20]$ at the LHC, the selection requirements are optimized separately for leptonic and fully hadronic final states from the top quark decays. The data set used corresponds to an integrated luminosity of $20.3 \mathrm{fb}^{-1}$ of proton-proton collisions at a center-of-mass energy of $8 \mathrm{TeV}$ and $4.5 \mathrm{fb}^{-1}$ at $7 \mathrm{TeV}$ recorded by the ATLAS detector. A search for the SM Higgs boson produced in association with a pair of top quarks, $\mathrm{t} \overline{\mathrm{t}} \mathrm{H}$, is presented using $20.3 \mathrm{fb}^{-1}$ of pp collision data at $\sqrt{s}=8 \mathrm{TeV}$, collected with the ATLAS detector for $\mathrm{H} \rightarrow \mathrm{b} \bar{b}$ decay and in addition to the $8 \mathrm{TeV}$ data, $4.5 \mathrm{fb}^{-1}$ of $7 \mathrm{TeV}$ data for $\mathrm{H} \rightarrow \gamma \gamma$. The search designed for the $\mathrm{H} \rightarrow \mathrm{b} \overline{\mathrm{b}}$ decay mode uses events containing one or two electrons or muons. The Higgs boson is assumed to have a mass of $125 \mathrm{GeV}$. In or- 

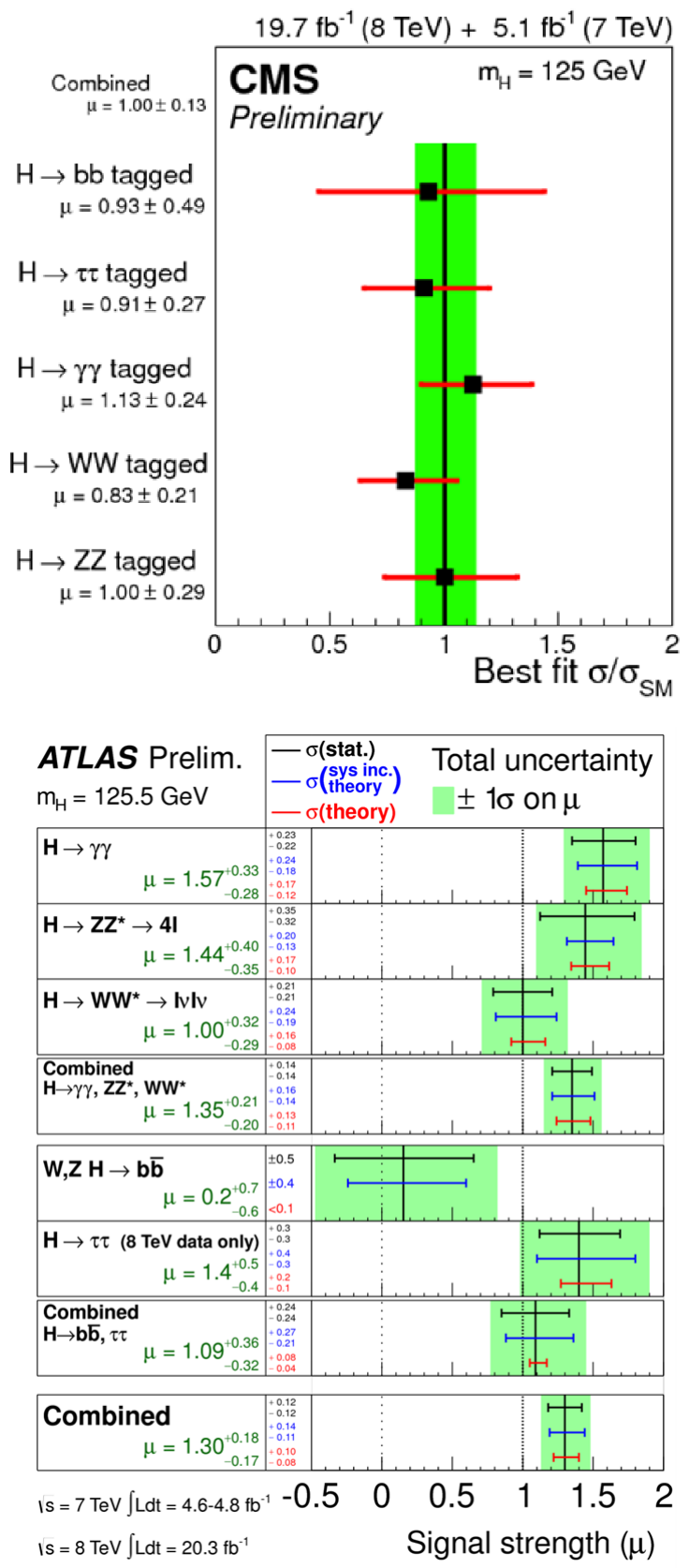

Figure 9. Best-fit values of the signal strength $\mu=\sigma / \sigma[S M]$, for the combination in solid vertical line and for individual channels in CMS (top) [23] and ATLAS (bottom) [21].

der to improve the sensitivity of the search, events are categorized according to their jet and b-tagged jet multiplicities. A multivariate technique is used to discriminate between signal and background events, which are dominated by $t \bar{t}+$ jets production. By performing a fit, allowing for an arbitrary Higgs boson signal strength, the ratio of the measured signal strength to the SM expectation at Higgs boson mass of $125.4 \mathrm{GeV}$ is found to be $\mu=1.7_{-1.1}^{1.3}$. A search for the SM Higgs boson produced in association with a top-quark pair (tțH) is presented, using data samples corresponding to integrated luminosities of up to $5.1 \mathrm{fb}^{-1}$ and $19.7 \mathrm{fb}^{-1}$ collected by the CMS detector in pp collisions at center-of-mass energies of 7 and $8 \mathrm{TeV}$ respectively. The
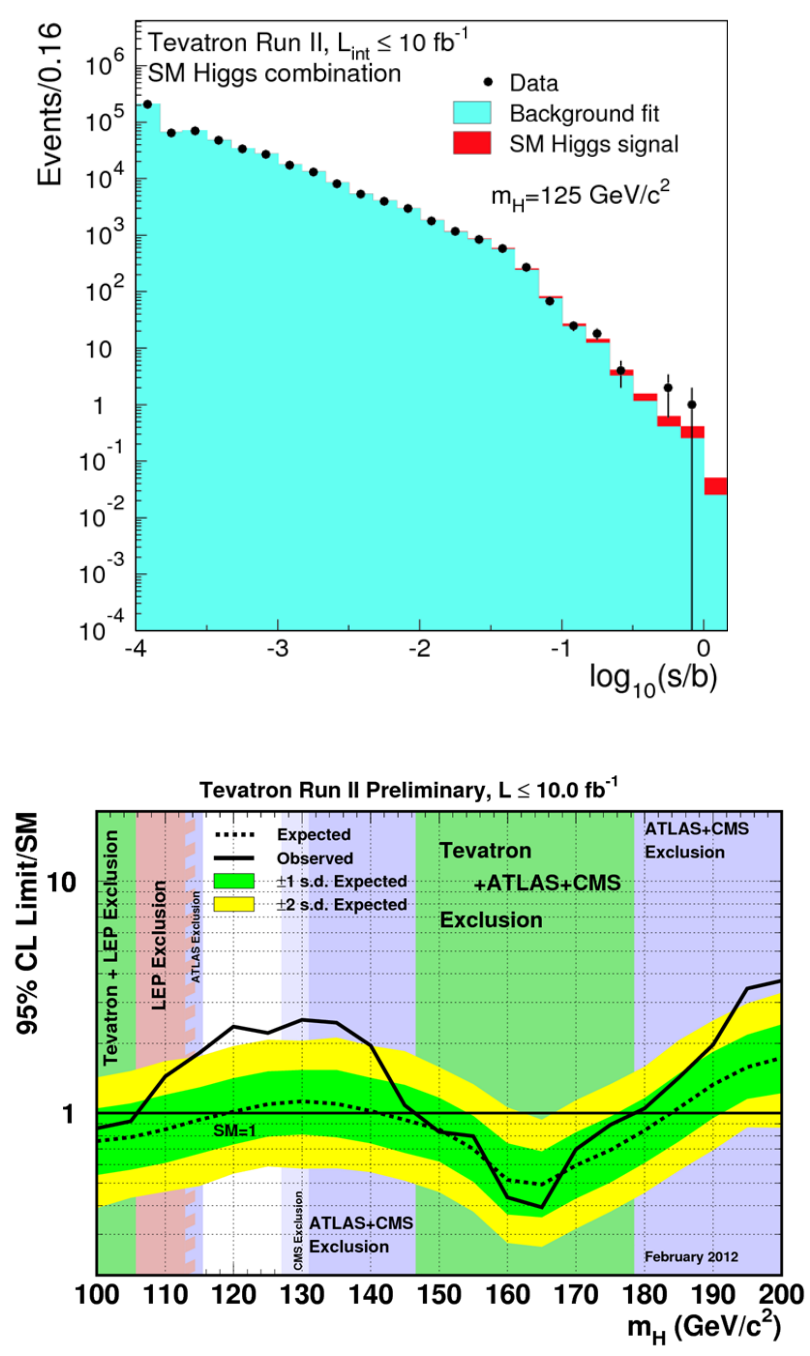

Figure 10. Distribution of $\log (\mathrm{s} / \mathrm{b})$, for the data from all contributing Higgs boson search channels from CDF and DØ, for $\mathrm{m}_{\mathrm{H}}=125 \mathrm{GeV}$ (top); observed and expected (median, for the background-only hypothesis) $95 \%$ C.L. upper limits on the ratios to the SM cross section, as functions of the Higgs boson mass for the combined CDF and DØ analyses (bottom) [24, 25].

search is based on the following signatures of the Higgs boson decay: $\mathrm{H}$ to hadrons, $\mathrm{H}$ to photons and $\mathrm{H}$ to leptons. The results are characterized by an observed $\mathrm{t} \overline{\mathrm{t}} \mathrm{H}$ signal strength relative to the SM cross section, $\mu=\sigma / \sigma[S M]$, under the assumption that the Higgs boson decays as expected in the standard model. The best fit value obtained by the CMS experiment is $\mu=2.8 \pm 1.0$ for a Higgs boson mass of $125.6 \mathrm{GeV}$.

\subsection{Combination Results at LHC and Tevatron}

The measurement of the combination of different Higgs boson decay channels using the full Run-I collision data sample recorded by the ATLAS experiment at the LHC for the channels $\mathrm{H} \rightarrow \gamma \gamma, \mathrm{H} \rightarrow \mathrm{ZZ}^{*} \rightarrow 4 \ell, \mathrm{H} \rightarrow \mathrm{WW}^{*} \rightarrow \ell \nu \ell v$, $\mathrm{H} \rightarrow \mathrm{b} \bar{b}$ and $\mathrm{H} \rightarrow \tau \tau$ have been presented [21]. The combined signal strength is determined to be $\mu=1.30 \pm$ 0.12 (stat) ${ }_{-0.11}^{+0.14}$ (sys) at a mass of $125.5 \mathrm{GeV}$. Evidence for 

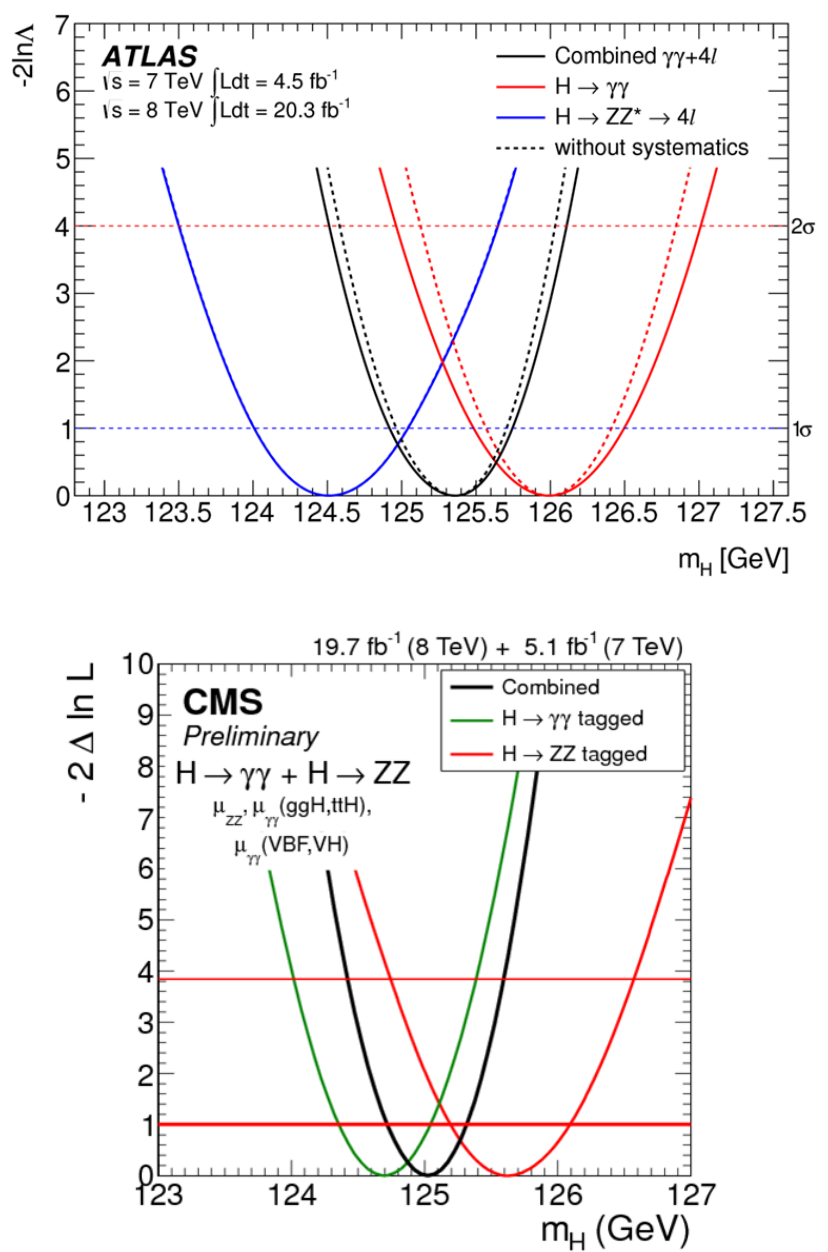

Figure 11. Scans of the test statistic $q\left(\mathrm{~m}_{\mathrm{H}}\right)$ versus the Higgs boson mass $\mathrm{m}_{\mathrm{H}}$ for the combination of the $\mathrm{H} \rightarrow \gamma \gamma$ and $\mathrm{H} \rightarrow \mathrm{ZZ}^{*} \rightarrow 4 \ell$ channels in ATLAS (top) [22] and CMS (bottom) [23].

direct decay into fermions is found at the $3.7 \sigma$ level from the combination of the $\mathrm{H} \rightarrow \mathrm{b} \overline{\mathrm{b}}$ and $\mathrm{H} \rightarrow \tau \tau$ channels with $\mathrm{a}$ signal strength of $\mu_{b b, \tau \tau}=1.09 \pm 0.24$ (stat) ${ }_{-0.21}^{+0.27}$ (sys). A comprehensive set of Higgs boson production and decay measurements are combined [23] by the CMS experiment at the LHC. The decays to $\gamma \gamma, \mathrm{ZZ}, \mathrm{WW}, \tau \tau$, and bb pairs are exploited, including studies targeting Higgs bosons produced in association with a pair of top quarks. The entire Run-I data sample has been used and the final detector calibration and alignment are used in the event reconstruction. For the measured mass combining the high resolution bosonic decay modes, the event yields obtained in the different analyses tagging specific decay modes and production mechanisms are consistent with those expected for the standard model Higgs boson. The combined best-fit signal strength, relative to the standard model expectation, is found to be $1.00 \pm 0.09$ (stat.) ${ }_{-0.07}^{+0.08}$ (theo.) \pm 0.07 (syst.) at the measured mass. Various searches for deviations in the magnitudes of the Higgs boson scalar couplings from those predicted for the standard model are performed with no significant deviations found. Combining tau pair and bottom quark pair decay modes at CMS as seen in Figure 9, the significance for Higgs boson decay to fermions

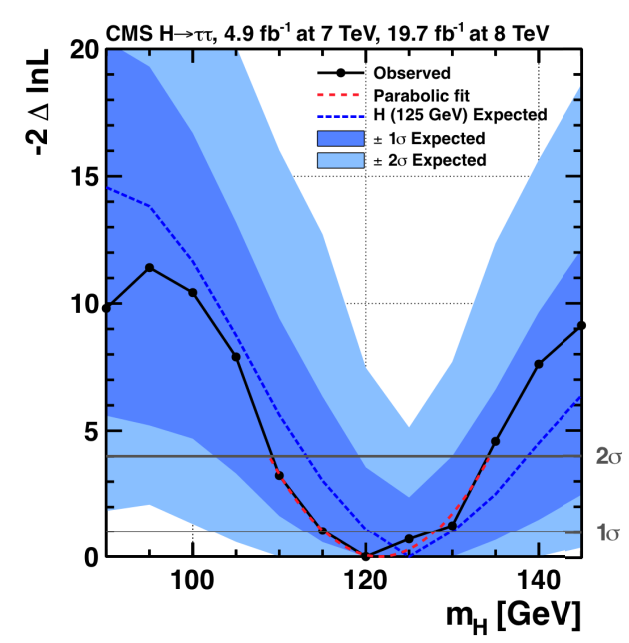

Figure 12. The measured mass from log-likelihood scan in the di-tau channel at CMS [14].

at $125 \mathrm{GeV}$ is observed to be $3.8 \sigma$ showing a strong direct evidence of Higgs-fermion coupling [15] at the LHC.

At the Tevatron with up to $10 \mathrm{fb}^{-1}$ of $\mathrm{p} \overline{\mathrm{p}}$ collision data, the combination of all search channels [24, 25] for $\mathrm{m}_{\mathrm{H}}=125 \mathrm{GeV}$ with the background subtracted distribution of the final discriminant for all channels are sorted as a function of $\mathrm{s} / \mathrm{b}$ and then added. An excess of events in the highest $\mathrm{s} / \mathrm{b}$ bins is observed and can be visualized in Figure 10. A significant signal-like excess in the mass range between 115 and $140 \mathrm{GeV}$ is observed. The background pvalue of that excess corresponds to $3.0 \sigma$ for $\mathrm{m}_{\mathrm{H}}=125 \mathrm{GeV}$. That excess arises from both CDF and DØ data, as expected from the presence of a standard model Higgs boson of mass $125 \mathrm{GeV}$. The results are interpreted in terms of limits on the Higgs boson production measured in units of the expected SM production as a function of the Higgs boson mass. The combined CDF and DØ results have almost reached the exclusion sensitivity for the full mass range $[90,185] \mathrm{GeV}$ for the SM Higgs boson search.

\section{Higgs Boson Properties Measurement}

\subsection{Mass}

The LHC experiments, ATLAS and CMS, have the ability of a very precise measurement of the Higgs boson mass due to the significant relevance of the high resolution boson decay modes $\mathrm{H} \rightarrow \gamma \gamma$ and $\mathrm{H} \rightarrow \mathrm{ZZ}^{*} \rightarrow 4 \ell$ with an excellent invariant mass resolution of $1-2 \%$ in the mass range of interest. The measurement of the mass of the discovered boson in the $\mathrm{H} \rightarrow \mathrm{ZZ}^{*} \rightarrow 4 \ell$ channel relied largely on the maximization of the efficiency for low transverse momentum leptons and on the precise calibration of the lepton momentum scale and resolution using $\mathrm{J} / \Psi, \Upsilon$ and $\mathrm{Z}$ boson decays to di-leptons. In the case of the $\mathrm{H} \rightarrow \gamma \gamma$ channel, the mass measurement depended largely both on the possibility to maintain a good mass resolution in high pileup regime (from both the energy and the direction contribution) and on the understanding of the electron/photon ex- 

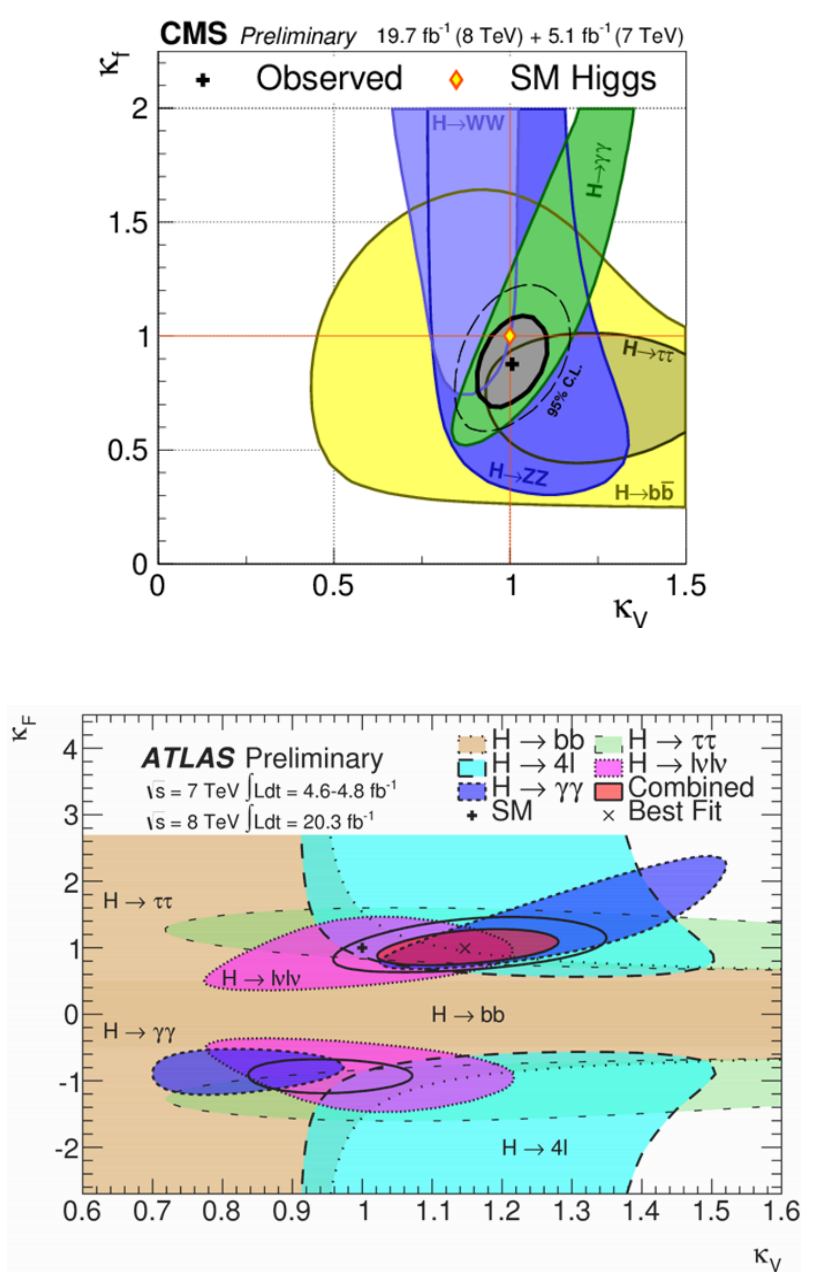

Figure 13. The $68 \%$ CL contours for individual channels (colored swaths) and for the overall combination (thick curve) for the $\left(\kappa_{\mathrm{V}}, \kappa_{\mathrm{f}}\right)$ parameters with the global best-fit values and the SM expectation of $\left(\kappa_{\mathrm{V}}, \kappa_{\mathrm{f}}\right)=(1,1)$ indicated from the CMS (top) [23] and ATLAS (bottom) [21] experiments.

trapolation for the energy scale (depending on the detector material, the shower shapes, etc). Once the two most sensitive channels were combined by ATLAS and CMS, the best fit values for the mass of the discovered boson remained consistent with each other within the uncertainties. In the CMS experiment, from the high resolution $\gamma \gamma$ and ZZ channels, the mass of the Higgs boson [23] is measured to be $125.03_{-0.27}^{+0.26}$ (stat.) ${ }_{-0.15}^{+0.13}$ (syst.) $\mathrm{GeV}$, with the precision dominated by the statistical uncertainty. In the ATLAS experiment as shown in Figure 11, the mass of the Higgs boson [22] is measured to be $125.36 \pm 0.37$ (stat.) \pm 0.18 (syst.) $\mathrm{GeV}$ with the precision dominated by the statistical uncertainty again. The mass of the Higgs boson measured in the tau pair decay mode at the CMS experiment is $122 \pm 7 \mathrm{GeV}$ obtained from a parabolic fit of the log-likelihood scan of the observed mass points in data as shown in Figure 12.

\subsection{Coupling}

The compatibility of the measured yields at the LHC for the studied channels with the predictions for the SM

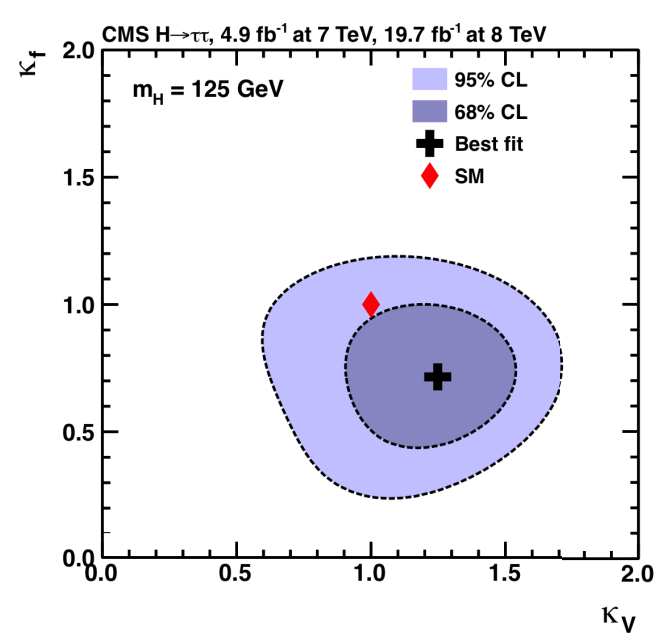

Figure 14. 2-D scan of couplings to vector boson and fermion scaled to the SM expectation at CMS in di-tau channel [14].

Higgs boson is tested under various benchmark assumptions probing salient features of the couplings [21, 23]. The measurements of the signal strengths for different decay modes do not give direct information on the relative contributions of the different production mechanisms. Furthermore, fixing the ratios of the production cross sections for the various processes to the values predicted by the standard model may conceal differences between data and theoretical predictions. Therefore, in addition to the signal strengths of different decay channels, the signal strengths of different production processes contributing to the same decay channel are determined, exploiting the sensitivity offered by the use of event categories in the analyses of all the channels. The data are fitted separating the VBF and $\mathrm{VH}$ processes, which involve the Higgs boson coupling to vector bosons, from the $\mathrm{ggF}$ and $\mathrm{ttH}$ processes, which involve the Higgs boson coupling to fermions (mainly the top-quark). The framework for the measurement is made under the assumption that the signals observed in the different search channels originate from a single narrow resonance with a mass near $125.5 \mathrm{GeV}$. The case of several, possibly overlapping, resonances in this mass region is not considered. The width of the assumed Higgs boson near $125.5 \mathrm{GeV}$ is neglected, i.e. the zero-width approximation is used. Only modifications of couplings strengths, i.e. of absolute values of couplings, are taken into account, while the tensor structure of the couplings is assumed to be the same as in the SM. This means in particular that the observed state is assumed to be a CP-even scalar as in the SM. The LO-motivated coupling scale factors $\kappa_{j}$ are defined in such a way that the cross section $\sigma_{j}$ and the partial decay width $\Gamma_{j}$ associated with the SM particle $j$ scale with the factor $\kappa_{j}^{2}$ when compared to the corresponding SM prediction. Measurements of production and decay mode specific signal strengths and Higgs boson coupling determinations in different benchmark models show good agreement with the standard model Higgs boson hypothesis. The couplings of the Higgs boson to vector bosons and fermions scaled to the SM expectations, has been shown 


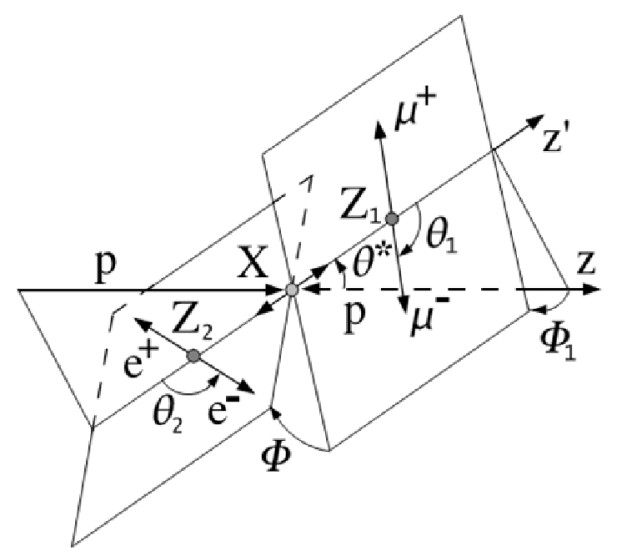

Figure 15. The decay topology of the Higgs boson in the 4lepton configuration used to construct the MELA discriminant.

by ATLAS and CMS in Figure 13. Compatibility with the SM is found in all the tests performed, with probabilities ranging from $7 \%$ to $21 \%$. In the tau pair decay channel [14], the measured couplings of the Higgs boson by the CMS experiment to vector bosons and fermions scaled with respect to SM, show consistency within around one standard deviations from SM predictions as shown in Figure 14 , where the $\mathrm{H} \rightarrow \mathrm{WW}$ process is added as a signal.

\subsection{Spin / Parity}

Both ATLAS and CMS collaborations used a similar approach to probe the spin and parity of the discovered particle $[11,12]$; the angular distributions of the decay products of the particle can in fact give information about its spin and parity. A matrix element likelihood approach (MELA) [26] was introduced for the $\mathrm{H} \rightarrow \mathrm{ZZ}^{*} \rightarrow 4 \ell$ analysis based on the probability ratio of the signal and background hypotheses, $\mathrm{K}_{\mathrm{D}}=\mathrm{P}_{\text {sig }} /\left(\mathrm{P}_{\text {sig }}+\mathrm{P}_{\text {bkg }}\right)$, where the leading-order matrix elements define the probabilities for each value of $\mathrm{m}_{4 \ell}$ incorporating the masses of dilepton pairs and five angles fully defining a four-lepton configuration in their center-of-mass frame as seen in Figure 15 for the construction of the kinematic discriminant. The discriminant is constrained to be between zero and one, and the relative normalization of probabilities is chosen to equate probabilities for signal and background distributions above and below 0.5 , respectively. In a similar way, the $0^{+}$scalar state is treated as a signal and the hypothesis under test, for example pseudoscalar $0^{-}$state, as the background to measure the spin and parity of the Higgs boson in the $\mathrm{H} \rightarrow \mathrm{ZZ}^{*} \rightarrow 4 \ell$ channel using the MELA discriminant. Test of spin and parity hypotheses were performed by using the CLs statistical approach. First the compatibility of data with the $0^{+}$hypothesis for the SM Higgs boson was tested. Then, alternative hypotheses were tested assuming the most general scattering amplitude for different spin and parity combinations. CMS used the $\mathrm{H} \rightarrow \mathrm{ZZ}^{*} \rightarrow 4 \ell$ analysis to test several spin/parity hypotheses corresponding to pure states expected in different models other than the SM. Its spin-parity properties are found to be consis-
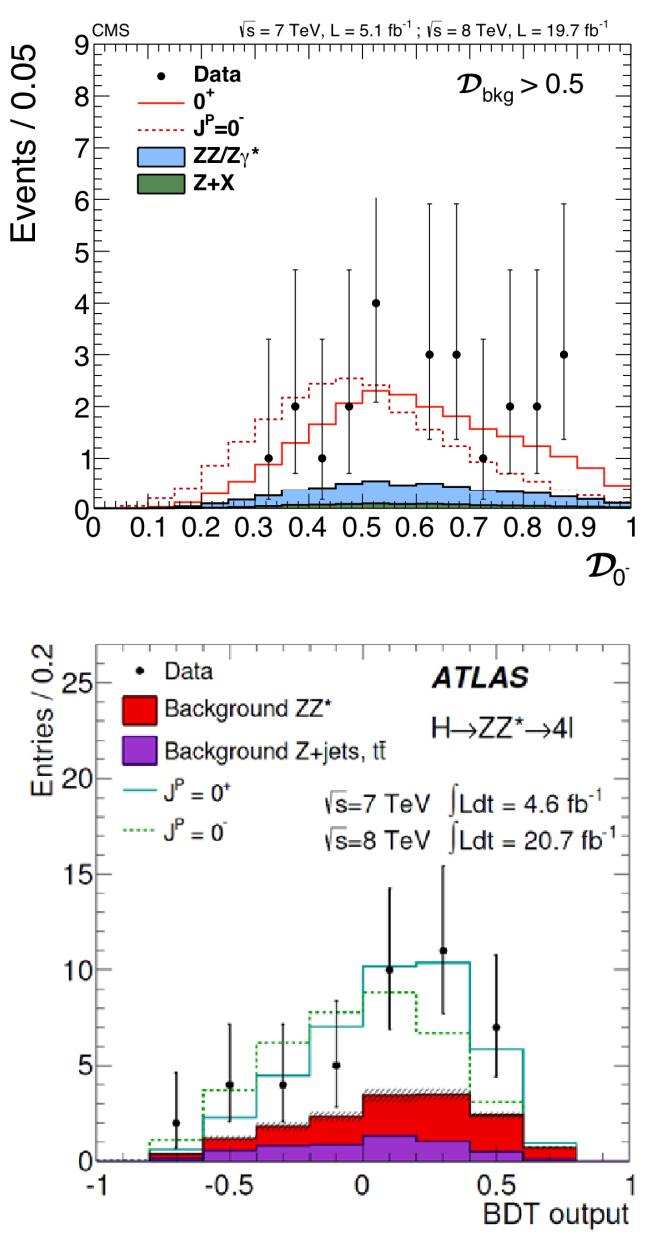

Figure 16. Distribution of the $\mathrm{D}^{\mathrm{JP}}$ discriminant shown for data and expectation for scalar and pseudoscalar $\mathrm{J}^{\mathrm{P}}$ states in CMS (top) [12]; and multivariate discriminant output incorporating the 4-lepton decay topology shown in data and expectation for scalar and pseudoscalar $\mathrm{J}^{\mathrm{P}}$ states in ATLAS (bottom) [11].

tent with the expectations for the standard model scalar Higgs boson. The hypotheses of a pseudoscalar and all tested spin-one boson hypotheses are excluded at a $99 \%$ confidence level or higher. All tested spin-two boson hypotheses are excluded at a $95 \%$ confidence level or even higher. ATLAS has also reported similar results favouring the scalar nature of the Higgs boson. In order to distinguish between pairs of spin and parity states, the reconstructed observables, namely the five angles and the two invariant masses, are combined using a multivariate discriminant based on a boosted decision tree. The BDT is trained on simulated signal events after full reconstruction and event selection. Dedicated discriminants are defined for the separation between the standard model $\mathrm{J}^{\mathrm{P}}=0^{+}$ hypothesis and each of the considered alternative models, $\mathrm{J}^{\mathrm{P}}=0^{-}, 1^{+}, 1^{-}, 2^{+}$. In the case of the spin- 2 hypothesis, the studies are performed as a function of the quark-antiquark production fraction. The response of the BDT classifiers is evaluated separately for each pair of signal hypotheses, including the expected backgrounds from other SM processes. The ATLAS data favours the spin-0 nature of the Higgs boson, with positive parity being strongly preferred 
over the pseudoscalar nature as shown in Figure 16, and the other tested spin/parity hypotheses being disfavoured.

\subsection{Decay Width}

It has recently been proposed [27] to constrain the Higgs boson width using its off-shell production and decay to two $\mathrm{Z}$ bosons away from the resonance peak [28]. In the dominant gluon fusion production mode, the off-shell production cross section is known to be sizable. This arises from an enhancement in the decay amplitude from the vicinity of the $\mathrm{Z}$ boson pair production threshold. A further enhancement comes, in gluon fusion production, from the top quark pair production threshold. The zero-width approximation is inadequate and the ratio of the off-shell cross section above $2 \mathrm{~m}_{\mathrm{Z}}$ to the on-shell signal is of the order of $8 \%[28,29]$. A measurement of the relative offshell and on-shell production in the $\mathrm{H} \rightarrow \mathrm{ZZ}$ channel provides direct information on $\Gamma_{\mathrm{H}}$, as long as the coupling ratios remain unchanged, i.e. the gluon fusion production is dominated by the top quark loop and there are no new particles contributing. In particular, the on-shell production cross section is unchanged under a common scaling of the squared product of the couplings and of the total width $\Gamma_{\mathrm{H}}$, while the off-shell production cross section increases linearly with this scaling factor.

The measurement of the $\mathrm{ZZ}$ final state in the mass range above the $2 \mathrm{~m}_{\mathrm{Z}}$ threshold provides a unique opportunity to measure the off-shell coupling strength of the observed Higgs boson. Constraints on the total Higgs boson width [31] have been placed by the CMS experiment using its relative on-shell and off-shell production and decay rates to 4 leptons or 2 leptons and 2 neutrinos. The results presented by CMS is based on the full Run-I pp collision data set. The four-lepton analysis uses the measured invariant mass distribution near the peak and above the Z-boson pair production threshold, as well as a likelihood discriminant to separate the gluon fusion $\mathrm{ZZ}$ production from the $\mathrm{q} \overline{\mathrm{q}} \rightarrow \mathrm{ZZ}$ background, while the two-lepton plus two-neutrino off-shell analysis relies on the transverse mass distribution. The presented analysis determines the independent contributions of the gluon fusion and VBF production mechanisms from the data in the on-shell region. It relies nevertheless on the knowledge of the coupling ratios between the off-shell and on-shell production, i.e. the dominance of the top quark loop in the gluon fusion production mechanism and the absence of new particle contribution in the loop. The presence of anomalous couplings in the HVV interaction would lead to enhanced off-shell production and would make our constraint tighter. The combined fit of the $4 \ell$ and $2 \ell 2 v$ channels leads to an upper limit on the Higgs boson width of $\Gamma_{\mathrm{H}}<22$ $\mathrm{MeV}$ at a $95 \%$ confidence level, which is 5.4 times the expected width of the SM Higgs boson as seen in Figure 17. This result improves by more than two orders of magnitude upon previous experimental constraints on the new boson decay width from the direct measurement at the resonance peak. A determination of the off-shell Higgs boson signal strength in the $\mathrm{ZZ} \rightarrow 4 \ell$ and $\mathrm{ZZ} \rightarrow 2 \ell 2 \mathrm{v}$

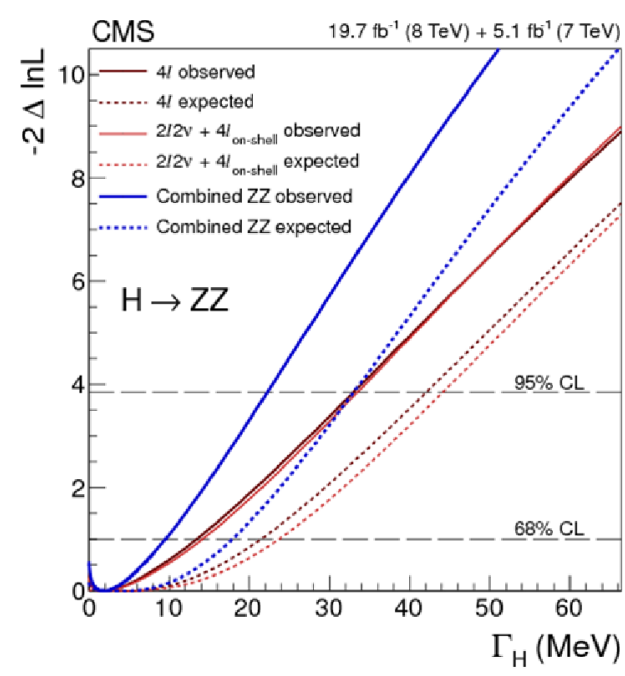

Figure 17. Likelihood scan versus the decay width $\Gamma_{\mathrm{H}}$ variable at the CMS experiment [31].

final states has also been presented by the ATLAS experiment, from which limits on the decay width [30] of the Higgs boson have been derived, using pp collision data corresponding to an integrated luminosity of $20.3 \mathrm{fb}^{-1}$ at a collision energy of $\sqrt{s}=8 \mathrm{TeV}$. The $95 \%$ confidence level upper CLs limit on the off-shell signal strength $\mu_{\text {off-shell }}$ is in the range 5.6-9.0 when varying the unknown $\mathrm{gg} \rightarrow \mathrm{ZZ}$ background K-factor from higher-order QCD corrections between half and twice the known signal K-factor, with an expected range of 6.6-10.7. Assuming no energy scale dependence of the relevant Higgs boson couplings, a combination with the on-shell measurement of $\mu_{\text {on-shell }}$ in the $\mathrm{H} \rightarrow \mathrm{ZZ} \rightarrow 4 \ell$ channel yields an observed (expected) $95 \%$ C.L. upper limit on $\Gamma_{\mathrm{H}} / \Gamma_{\mathrm{H}}^{\mathrm{SM}}$ in the range 4.8-7.7 (7.0-12.0) under the same variations of the background $\mathrm{K}$-factor.

\subsection{Cross section}

The measurements of fiducial and differential cross sections [32] have been presented for Higgs boson production in proton-proton collisions at a center-of-mass energy of $\sqrt{s}=8 \mathrm{TeV}$ at the LHC. The analysis is performed in the $\mathrm{H} \rightarrow \gamma \gamma$ decay channel using $20.3 \mathrm{fb}^{-1}$ of data recorded by the ATLAS experiment. The signal is extracted using a fit to the diphoton invariant mass spectrum assuming that the width of the resonance is much smaller than the experimental resolution. The signal yields are corrected for the effects of detector inefficiency and resolution. The $\mathrm{pp} \rightarrow \mathrm{H} \rightarrow \gamma \gamma$ fiducial cross section is measured to be $43.2 \pm 9.4$ (stat.) ${ }_{-2.9}^{+3.2}$ (syst.) \pm 1.2 (lumi.) fb for a Higgs boson of mass $125.4 \mathrm{GeV}$ decaying to two isolated photons that have transverse momentum greater than $35 \%$ and $25 \%$ of the diphoton invariant mass and each with absolute pseudorapidity less than 2.37 in the detector. Four additional fiducial cross sections and two cross section limits are presented in phase space regions that test the theoretical modelling of different Higgs boson production mechanisms, or are sensitive to physics beyond the SM. 
Differential cross sections have also been measured, as a function of variables related to the diphoton kinematics and the jet activity produced in the Higgs boson events. The observed spectra are statistically limited but broadly in line with the theoretical expectations. The most accurate theoretical predictions for Higgs boson production via gluon fusion in the baseline fiducial region are calculated at the parton level. Higgs boson production via gluon fusion is simulated at next-to-leading-order (NLO) accuracy in QCD using the Powheg Box, with the CT10 parton distribution function (PDF). The LHC Higgs cross section working group recommends using a calculation for the cross section of Higgs boson production via gluon fusion that is accurate to next-to-next-leading-order (NNLO) + next-to-next-leading-logarithm (NNLL) in QCD and incorporates NLO electroweak corrections. The predictions are provided with uncertainties associated with renormalization, factorization and resummation scale variation, as well as an uncertainty from PDF variation. These predictions are corrected to the particle level to allow comparison to data, using diphoton acceptance, photon isolation and non-perturbative correction factors. The diphoton acceptance and photon isolation correction factors account for the decay of the Higgs boson to two isolated photons in the geometrical acceptance of the detector. They are determined using Powheg + Pythia 8 events with associated uncertainties from PDF and renormalization/factorization scale variations. The non-perturbative correction factors account for the impact of hadronization and underlying event activity. For events containing one or more jets, a parton-level cross section has been calculated for Higgs boson production via gluon fusion using soft-collinear effective theory, by combining NNLO + NNLL 0-jet and $\mathrm{NLO}+$ next-to-leading-logarithm (NLL) 1-jet cross sections. A sample of $\mathrm{H}+1$-jet events is produced at NLO accuracy in QCD using the Powheg Box, with the Minlo feature applied to include $\mathrm{H}+0$-jet events at NLO accuracy and interfaced to Pythia8 to produce fully hadronic final state and this sample is referred to as Minlo HJ. The data have been compared to the NLO + leading logarithm (LL) prediction provided by Minlo $\mathrm{HJ}$ for gluon fusion and the default MC samples for the other production mechanisms; the Minlo HJ prediction is normalised to the LHC cross section prediction using 1.54 as a $\mathrm{K}$-factor. For the differential distributions that probe the kinematics of the diphoton system, the HRES 2.2 calculation is used to provide the prediction for Higgs boson production via gluon fusion. HRES is accurate to NNLO + NNLL in QCD but does not contain any electroweak corrections. The uncertainty associated with missing higher orders in the calculation is derived from the envelope of cross-section predictions obtained by simultaneously varying the renormalization, factorization and resummation scales by a factor. The differential cross section as a function of the jet multiplicity has been calculated for both jets with $\mathrm{p}_{\mathrm{T}}>30 \mathrm{GeV}$ and $\mathrm{p}_{\mathrm{T}}>50 \mathrm{GeV}$. The agreement between theory and data is satisfactory with a non-significant excess of events in data at the highest jet multiplicities as seen in Figure 18. Thus, in addition to the fiducial cross sections, twelve dif-
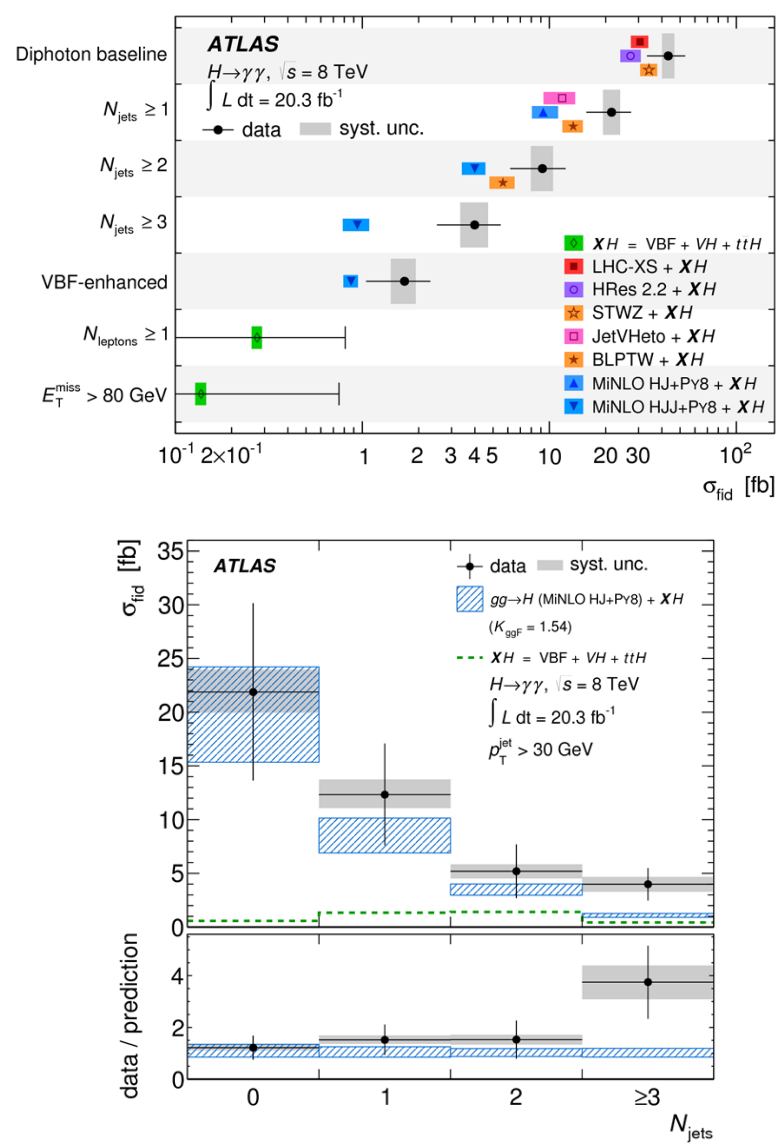

Figure 18. The ATLAS measurement of the fiducial cross section (top) and differential cross section in bins of jet multiplicity sensitive to the Higgs boson production modes (bottom) in the $\mathrm{H} \rightarrow \gamma \gamma$ channel [32].

ferential cross sections were measured within the baseline fiducial volume defined by the kinematics of the two photons. Collectively, these measurements probe the Higgs boson kinematics, the jet activity produced in association with the Higgs boson, and the prevalence of vector boson fusion, as well as the spin, charge conjugation and parity nature of the Higgs boson. In all cases, the data have been found to be in agreement with the SM expectations.

\section{Conclusion}

Both the ATLAS and CMS experiments reported discovery of a new boson in their SM Higgs boson searches with the 2011 and 2012 pp collision data at the LHC in the high resolution boson decay modes of $\gamma \gamma$ and $\mathrm{ZZ}^{*} \rightarrow 4 \ell$. Subsequent measurements from the two experiments have found the coupling properties and the spin-CP nature of the new boson compatible with the expectations of the SM Higgs boson, which confirm that the new boson is a Higgs boson. A broad excess of events is observed at the LHC for the SM Higgs boson search in the tau pair decay mode consistent with the $125 \mathrm{GeV}$ Higgs boson signal from high resolution boson decay modes providing the first direct indication of the Higgs boson coupling to leptons. Combining tau pair and bottom quark pair decay modes by the 
CMS experiment, the significance for Higgs boson decay to fermions at $125 \mathrm{GeV}$ is $3.8 \sigma$ showing a direct evidence of Higgs-fermion coupling at the LHC. The mass of the Higgs boson measured in the di-tau channel by the CMS experiment is $122 \pm 7 \mathrm{GeV}$ from a parabolic fit of the likelihood scan of the Higgs mass. The decay width of the Higgs boson has been constrained to less than $22 \mathrm{MeV}$ at a $95 \%$ confidence level at the LHC by the CMS experiment, which is 5.4 times the expected width of the SM Higgs boson. Measurements of the Higgs boson fiducial and differential cross sections have also been made at the LHC by the ATLAS experiment. In the Run-2 LHC, the measurement of properties of the Higgs boson in different decay channels would continue looking for deviations from SM as well as the presence of other Higgs-like states.

At the Tevatron with up to $10 \mathrm{fb}^{-1}$ of $\mathrm{p} \overline{\mathrm{p}}$ collision data, the SM Higgs boson is excluded at 95\% C.L. from 90 to $109 \mathrm{GeV}$ and 149 to $182 \mathrm{GeV}$. The expected exclusion regions in the absence of a signal are 90-120 GeV and 140-184 GeV. The results of the Higgs boson decaying to bottom quarks were validated through a measurement of the diboson $(\mathrm{WZ}+\mathrm{ZZ})$ production cross section using the same data samples and analysis techniques, treating those diboson processes as signal. The resulting diboson cross section measurement is in agreement with the SM prediction. A significant excess of events is observed in the mass range between 115 and $140 \mathrm{GeV}$. The local significance at $\mathrm{m}_{\mathrm{H}}=125 \mathrm{GeV}$ corresponds to $3.0 \sigma$, with a median expected significance, assuming the SM Higgs boson is present at $\mathrm{m}_{\mathrm{H}}=125 \mathrm{GeV}$, of $1.9 \sigma$, with a best-fit signal strength of $1.44_{-0.56}^{+0.59}$ times the SM expectation.

\section{References}

[1] The ATLAS Collaboration, Phys. Lett. B 716, 1 (2012) and all references therein.

[2] The CMS Collaboration, Phys. Lett. B 716, 30 (2012) and all references therein.

[3] The ATLAS Collaboration, JINST 3, S08003 (2008).

[4] The CMS Collaboration, JINST 3, S08004 (2008).

[5] The D0 Collaboration, Nucl. Instrum. Meth. A 565, 463 (2006).

[6] The CDF Collaboration, J. Phys. G 34, 2457 (2007).

[7] The ATLAS Collaboration, arXiv:1408.7084 [hepex] and all references therein.

[8] The ATLAS Collaboration, ATLAS-CONF-2013012.
[9] The CMS Collaboration, arXiv:1407.0558 [hep-ex] and all references therein.

[10] The ATLAS Collaboration, arXiv:1408.5191 [hepex] and all references therein.

[11] The ATLAS Collaboration, Phys. Lett. B 726, 120 (2013).

[12] The CMS Collaboration, Phys. Rev. D 89, 092007 (2014) and all references therein.

[13] The ATLAS Collaboration, ATLAS-CONF-2013108 and all references therein.

[14] The CMS Collaboration, JHEP 05, 104 (2014) and all references therein.

[15] The CMS Collaboration, Nat. Phys. 10, 557 (2014) and all references therein.

[16] The D0 and CDF Collaborations, Phys. Rev. Lett. 109, 071804 (2012) and all references therein.

[17] The CDF Collaboration, Phys. Rev. Lett. 109, 111802 (2012) and all references therein.

[18] The D0 Collaboration, Phys. Rev. Lett. 109, 121802 (2012) and all references therein.

[19] The ATLAS Collaboration, ATLAS-CONF-2014043 and all references therein.

[20] The CMS Collaboration, arXiv:1408.1682 [hep-ex] and all references therein.

[21] The ATLAS Collaboration, ATLAS-CONF-2014009 and all references therein.

[22] The ATLAS Collaboration, Phys. Rev. D 90, 052004 (2014) and all references therein.

[23] The CMS Collaboration, CMS-PAS-HIG-14-009 and all references therein.

[24] The D0 and CDF Collaborations, Phys. Rev. D 88, 052014 (2013) and all references therein.

[25] The D0 and CDF Collaborations, arXiv:1203.3774 [hep-ex] and all references therein.

[26] The CMS Collaboration, Phys. Rev. Lett. 110, 081803 (2013).

[27] F. Caola and K. Melnikov, Phys. Rev. D 88, 054024 (2013).

[28] N. Kauer and G. Passarino, JHEP 08, 116 (2012).

[29] N. Kauer, Mod. Phys. Lett. A 28, 1330015 (2013).

[30] The ATLAS Collaboration, ATLAS-CONF-2014042 and all references therein.

[31] The CMS Collaboration, Phys. Lett. B 736, 64 (2014) and all references therein.

[32] The ATLAS Collaboration, arXiv:1407.4222 [hepex] and all references therein. 